\title{
Compression ultrasonography for diagnostic management of patients with clinically suspected deep vein thrombosis: prospective cohort study
}

\author{
Alberto Cogo, Anthonie W A Lensing, Maria M W Koopman, Franco Piovella, Sergio Siragusa, \\ Philip S Wells, Sabina Villalta, Harry R Büller, Alexander G G Turpie, Paolo Prandoni
}

\begin{abstract}
Objective: To evaluate the safety of withholding anticoagulant treatment from patients with clinically suspected deep vein thrombosis but normal findings on compression ultrasonography.

Design: Compression ultrasonography was done with a simplified diagnostic procedure limited to the common femoral vein in the groin and the popliteal vein extending down to the trifurcation of the calf veins. Patients with normal ultrasonography findings at presentation were retested 1 week later.

Main outcome measure: The incidence of venous thromboembolic complications during follow up for 6 months in patients in whom anticoagulant treatment was withheld on the basis of normal results on two ultrasonography tests 1 week apart.

Setting: University research centres in four hospitals.

Results: A total of 1702 patients were included in the study. Abnormal results on compression ultrasonography at presentation or at 1 week were found in 400 and 12 patients, respectively, for a prevalence of deep vein thrombosis of $24 \%$. None of the patients were lost to follow up. Venous thromboembolic complications during the week of serial testing occurred in a single patient and in eight patients during 6 months' follow up, resulting in a cumulative rate of venous thromboembolic complications of $0.7 \%$ (95\% confidence interval $0.3 \%$ to $1.2 \%$ ). The mean number of extra hospital visits and additional tests required per initially referred patient was 0.8 .

Conclusion: It is safe to withhold anticoagulant treatment from patients with clinically suspected deep vein thrombosis who have a normal result on compression ultrasonography at the time of presentation and at 1 week.
\end{abstract}

\section{Introduction}

Contrast venography is the standard method for the diagnosis of deep vein thrombosis, but its widespread use is hampered mainly due to the invasive nature of the test, limited availability, and the side effects associated with the use of contrast material. ${ }^{12}$ These limitations formed the basis for the development of non-invasive tests. Impedance plethysmography was the first well evaluated non-invasive method and had a high accuracy for proximal vein thrombosis in symptomatic patients but was less sensitive for thrombi confined to the calf veins. ${ }^{3}$ Therefore, impedance plethysmographic tests needed to be repeated several times to detect proximally extending thrombi in the calf vein. In early studies, impedance plethysmography was repeated five times over 2 weeks in patients who had an initially normal test result. ${ }^{4}$ In subsequent studies, the efficiency of the test procedure was further improved by reducing the number of repeat tests to three and later to two tests without compromising safety. ${ }^{6-8}$

Over the past decade, compression ultrasonography has emerged as the non-invasive method of choice for the evaluation of patients with clinically suspected deep vein thrombosis because of its excellent accuracy for deep vein thrombosis and wide availability. ${ }^{8-10}$ The procedure can be limited to a single spot in the groin and mid-popliteal fossa, where clear and reproducible images can be obtained. ${ }^{8-11}$ As is the case for impedance plethysmography, however, compression ultrasonography cannot accurately evaluate the calf veins. ${ }^{9}$ Consequently, serial compression ultrasonography testing remains necessary. To identify the relatively few patients whose compression ultrasonography result will convert from normal to abnormal, however, the test has to be repeated twice in all patients with an initial normal test result. ${ }^{8} 12$ This diagnostic approach is inconvenient for patients, labour intensive, and expensive. Recent observations showed that some symptomatic patients with normal results on compression ultrasonography in the mid-popliteal fossa have thrombi confined to the more distal part of the popliteal vein. ${ }^{11}{ }^{12}$ This could imply that an evaluation of the popliteal vein extending down to the trifurcation of the calf veins has the potential to increase sensitivity of the test at presentation necessitating only one repeat ultrasonography test 1 week later.

We evaluated the simplified ultrasound strategy in a large series of consecutive patients with clinically suspected deep vein thrombosis. The clinical validity of withholding anticoagulant treatment from patients who had normal results on compression ultrasonogra-
See editorial by

Davidson and Deppert

Istituto di

Semeiotica Medica, University of Padua, 35128 Padua, Italy

Alberto Cogo, research fellow Sabina Villalta, research fellow Paolo Prandoni, senior researcher

Policlinico San Matteo, Clinica Medica Seconda, 27100 Pavia, Italy

Franco Piovella, senior researcher Sergio Siragusa, research fellow

HGH McMaster Clinic, Hamilton General Hospital, Hamilton, Ontario L8L 2X2, Canada Philip S Wells, research fellow

Alexander G G Turpie, senior researcher

Centre for Vascular Medicine, Academic Medical Centre,

H-2, 1105 AZ

Amsterdam, Netherlands

Anthonie W A Lensing, senior researcher

Maria M W Koopman, senior researcher Harry R Büller, senior researcher

Correspondence to: Dr Lensing a.w.lensing@amc. uva.nl

BMJ 1998;316:17-20 
phy on presentation and on a single repeat test 1 week later was assessed during follow up for 6 months.

\section{Patients and methods}

\section{Patients}

All patients referred to one of the participating centres with a suspected first episode of deep vein thrombosis of the leg were potentially eligible for the study. Patients were excluded if they had had deep vein thrombosis in the past, had received anticoagulant treatment for more than 48 hours, had a contraindication to contrast venography, were under 18 years old, or lived too far from the study centre to return for follow up visits. In addition, patients who presented with concurrent symptoms of pulmonary embolism were excluded as symptomatic pulmonary embolism constituted one of the main study outcomes. Eligible patients who gave informed consent were enrolled. The study protocol was approved by the institutional review boards.

\section{Study design}

The objective of this study was to determine the clinical validity of normal compression ultrasound tests with a simplified examination limited to the common femoral vein and the popliteal vein extending down to the trifurcation of the calf veins. Patients with a normal result at presentation were not treated with anticoagulants and were scheduled for a single repeat test 1 week later which was performed with an identical approach as at presentation. If the repeat test also yielded normal results patients were considered not to have deep vein thrombosis and anticoagulant treatment was withheld. These patients were followed up for a period of 6 months and were asked to return to the study centre at 3 and 6 months or immediately if they had signs or symptoms of pulmonary embolism or deep vein thrombosis.

During follow up visits patients were questioned about their general health, recent hospital admissions, possible complications of venous thromboembolism, and use of anticoagulant treatment. Patients who did not return for follow up assessments were interviewed by telephone. Confirmatory testing (that is, contrast venography, perfusion-ventilation lung scanning, or pulmonary angiography) was planned in all patients with suspected venous thromboembolic complications. ${ }^{8}$ Necropsies were requested for all patients who died and in whom pulmonary embolism could not be excluded as the cause of death. An independent physician determined the cause of death if necropsy could not be performed. Most patients with abnormal ultrasonography findings had confirmatory venography to assess the positive predictive value.

\section{Ultrasonography technique}

The ultrasound examinations were performed with a 7.5-MHz linear array sonographic scanner (Advanced Technology Laboratories, ATL Ultramark 4, Bothell, Washington, United States). Only the common femoral and popliteal veins were evaluated. ${ }^{9}{ }^{10}$ With the patient in the supine position, the common femoral vein was visualised at the inguinal ligament by using the adjacent artery as a reference point. The popliteal vein was scanned with the patient in the prone or lateral decubitus position and the transducer placed posteriorly in the mid-popliteal fossa. Assessment of the proximal veins along their entire course in the thigh results in loss of specificity and is not indicated because symptomatic patients with venous thrombosis usually have large thrombi affecting at least the popliteal or common femoral vein. ${ }^{11}$ For the evaluation of the distal popliteal vein, the transducer was moved slowly from the popliteal fossa along the calf down to the trifurcation of the calf veins. No attempt was made to evaluate the calf veins. Failure to compress the lumen of the vein fully during compression with the ultrasound transducer was the sole criterion for the presence of deep vein thrombosis. ${ }^{9}$ Proximal deep vein thrombosis was excluded if both the common femoral and popliteal vein were fully compressible and no residual lumen was seen. Ultrasonic findings were scored as normal, abnormal, or inadequate for interpretation.

\section{Analysis}

The outcome measure was the total rate of symptomatic venous thromboembolic complications during follow up over 6 months defined as either a pulmonary embolism occurring before repeat ultrasonography or as pulmonary embolism or a deep vein thrombosis during long term follow up. The total complication rate was calculated with Kaplan-Meier survival analysis and was defined as 1 minus the complication free survival rate. This method takes into account the different numbers of patients at risk during the different time periods. An estimate of the exact $95 \%$ confidence interval was made with the $\mathrm{SD}$ as calculated by the Kaplan-Meier analysis.

It was estimated that about 1650 patients were needed to conclude with sufficient confidence that the clinical validity of our simplified compression ultrasound strategy was at least equivalent to that of the earlier non-invasive diagnostic strategies which used more repeat tests to exclude deep vein thrombosis. Feasibility was expressed as the mean number of extra visits to the hospital and the mean number of additional tests required per initially referred patient.

\section{Results}

During the recruitment period, 2113 consecutive outpatients with clinically suspected deep vein thrombosis were referred to the study centres for objective testing. Three hundred and seventy two patients $(17.6 \%)$ were excluded from the study: 175 with previous venous thrombosis, 77 had already started long term anticoagulant treatment, 52 because of geographic inaccessibility, 24 had contraindications to contrast material, 20 with clinically suspected pulmonary embolism at the time of referral, 15 were pregnant, five were aged less than 18 years, and in four ultrasonography could not be performed because of plaster cast or leg amputation. Thus, a total of 1741 patients were ruled eligible for the study, of whom 38 refused to give informed consent. Therefore, our analysis included 1703 patients, of whom 1046 (61\%) were women. The mean (range) age of the study subjects was 63.9 (18 to 96 ) years, and the median 
Summary of non-invasive diagnostic strategies used in patients with clinically suspected deep vein thrombosis

\begin{tabular}{|c|c|c|c|c|}
\hline Reference & Diagnostic strategy & $\begin{array}{c}\text { Prevalence of deep vein } \\
\text { thrombosis }(\%)\end{array}$ & $\begin{array}{c}\text { Complications during } 3 \text { month } \\
\text { follow up }(95 \% \mathrm{CI})\end{array}$ & $\begin{array}{c}\text { Mean No of extra hospital } \\
\text { visits/additional tests required per } \\
\text { patient }\end{array}$ \\
\hline \multicolumn{5}{|c|}{ Impedance plethysmography } \\
\hline Hull $1985^{4}$ & At days $1,2,3,5$ or $7,10,14^{*}$ & 29 & $1.8(0.2$ to 3.4$)$ & 3.9 \\
\hline Huisman $1986^{6}$ & At days $1,2,5,10$ & 30 & 0.7 (0 to 1.6$)$ & 2.1 \\
\hline Huisman $1989^{7}$ & At days $1,2,7$ & 45 & $0.8(0$ to 2.2$)$ & 1.3 \\
\hline Heijboer $1993^{8}$ & At day $1,2,8$ & 24 & $2.4(0.9$ to 4.0$)$ & 1.6 \\
\hline \multicolumn{5}{|c|}{ Compression ultrasonography } \\
\hline Sluzewski $1991^{12}$ & At days $1,2,7$ & 27 & $1.3(0$ to 3.9$)$ & 1.3 \\
\hline Heijboer $1993^{8}$ & At days $1,2,8$ & 21 & $1.5(0.3$ to 2.8$)$ & 1.6 \\
\hline Current study & At days 1,7 & 24 & 0.7 (0.3 to 1.2) & 0.8 \\
\hline
\end{tabular}

${ }^{*}$ Combined with iodine-125 fibrinogen leg scanning.

(range) interval between the onset of symptoms and the day of referral was 6 (1 to 60$)$ days.

\section{Ultrasonography}

Adequate compression ultrasonography results were obtained for 1702 of the 1703 consecutive patients. Of the $412(24 \%)$ patients with abnormal results, 400 were detected at presentation and 12 on repeat testing. Repeat testing was refused by 33 patients because signs and symptoms of deep vein thrombosis had disappeared. A total of 26 patients (23 at presentation and three on repeat testing 1 week later) had normal results for the common femoral vein and at the level of the mid-popliteal fossa but thrombosis was identified in the distal popliteal vein.

\section{Follow up}

Three patients presented before the scheduled repeat test at 1 week with clinically suspected pulmonary embolism; this was not confirmed by objective testing in two patients. The remaining patient, who initially presented with leg pain after calf muscle trauma, developed sudden shortness of breath on the fifth day after the initial normal result. The patient was admitted to a local hospital and received low dose subcutaneous standard heparin (5000 IU twice daily). No diagnostic investigations were performed. The next day the patient died, and necropsy revealed a massive pulmonary embolism. Eighteen patients returned before the scheduled 3 month follow up visits because of recurrent or aggravating symptoms of deep vein thrombosis of the leg (17 patients) or clinically suspected pulmonary embolism (one patient). Compression ultrasonography and venography confirmed the presence of deep vein thrombosis in seven patients and excluded deep vein thrombosis in 10 . Perfusionventilation lung scanning confirmed the presence of pulmonary embolism in the other patient. No suspected venous thromboembolic complications occurred after the 3 month follow up visit. The overall cumulative incidence of venous thromboembolic complications (that is, pulmonary embolism before repeat ultrasonography at 1 week and pulmonary embolism or deep vein thrombosis during long term follow up) was therefore $0.7 \%$ (95\% confidence interval $0.3 \%$ to $1.2 \%)$.

Twenty one patients died during the follow up over 6 months: cancer (11 patients), myocardial infarction (four), stroke (three), congestive heart failure (two), and liver cirrhosis (one). In 16 patients with normal results on ultrasonography at presentation the protocol was violated: anticoagulant treatment was initiated in seven patients and venography was performed in nine patients. Isolated calf vein thrombosis was found in one of these patients.

\section{Discussion}

Serial non-invasive testing for the diagnosis of symptomatic deep vein thrombosis is necessary because a proportion of patients with less extensive disease will have initial normal test results which may convert to abnormal on subsequent days. ${ }^{4-8}$ In the initial studies, a total of five tests were performed. Currently, three tests (at referral, the next day, and 1 week later) are required for optimal management.

The results of this study in a large series of patients with clinically suspected deep vein thrombosis indicate that compression ultrasound with a simplified examination is able both to identify most patients with deep vein thrombosis and to reduce safely the number of repeat tests to a single one with an interval of 1 week. Patients with an initial normal test result were not treated with anticoagulants and were scheduled for repeat testing 1 week later. Six months of follow up in patients with normal results showed a cumulative rate of venous thromboembolic complications of only $0.7 \%$; a finding which is at least equivalent to results observed in earlier studies that used serial impedance plethysmography or compression ultrasonography (table 1). ${ }^{6-8} 12$

In recent studies evaluating serial compression ultrasonography, two additional tests were needed to identify about $7 \%$ of the total number of patients with deep vein thrombosis. ${ }^{8}{ }^{12}$ With use of the simplified ultrasound examination only $3 \%$ of the total number of patients with deep vein thrombosis were identified by the single repeat test without observing an increased risk for venous thromboembolic complications at follow up. The extended evaluation of the popliteal vein down to the trifurcation of the calf veins resulted in the identification of small proximal deep vein thrombosis in an additional 26 patients, $6.3 \%$ of the total number of patients with confirmed venous thrombosis. The mean number of extra hospital visits and additional tests required per initially referred patients was 0.8 , which is considerably lower than that found in previous studies evaluating non-invasive diag- 
- Clinical diagnosis of suspected deep vein thrombosis is notoriously unreliable and objective diagnostic tests are indicated to confirm or refute the presence of this condition

- Ultrasonography with vein compressibility of the common femoral and popliteal vein is the non-invasive test of choice for the diagnostic management of patients with suspected deep vein thrombosis

- It is safe to withhold anticoagulant treatment from patients with suspected deep vein thrombosis who have normal results on compression ultrasonography on presentation and on a single repeat test 1 week later

- With the simplified compression ultrasound strategy the number of repeat tests can be safely reduced to a single test performed a week after presentation

- Most patients with deep vein thrombosis can be identified at presentation, making this strategy convenient to patients and less costly

nostic strategies for suspected deep vein thrombosis (table 1).

Although the use of the extended popliteal vein examination allowed for the earlier identification of patients with proximal vein thrombosis, the procedure resulted in more false positive test results. Thus, the positive predictive value for the assessment of the common femoral vein and the popliteal vein in the mid-popliteal fossa was $98.5 \%$, whereas this was $79 \%$ for the distal popliteal vein examination.

The reduction of the number of repeat ultrasound assessments to a single test 1 week later is clearly associated with lower costs but remains relatively cost ineffective as most patients with an initial normal result on ultrasonography do not have venous thrombosis. Although only 3\% (upper 95\% confidence limit 5\%) of patients with venous thrombosis were identified by the repeat test 1 week later, one can speculate whether this repeat test is actually needed. We consider that such an attractive option from the point of view of hospital logistics is still offset by the potential for fatal pulmonary embolism. Further refinements of the diagnostic strategy with the high negative predictive value of the D-dimer assay ${ }^{13}$ and the use of clinical decision rules, ${ }^{14}$ however, show promise.

We thank Drs L Vicentini, S Carbone, C Beltrametti, M Barone, and J Johnson for their technical help throughout the study.

Contributors: AWAL and PP designed the protocol, initiated and coordinated the study, analysed and interpreted the data, and are responsible for writing the paper. AC, FP, HRB, and AGGT were the local principal investigators who discussed core ideas and participated in the protocol design and execution of the study, particularly data documentation and quality control. They also edited the manuscript. MMWK, SS, PSW, and SV participated in the execution of the trial, particularly in assessing the patients for inclusion, performing ultrasound tests, and monitoring patients during follow up.

Funding: AWAL received a grant from the Dutch foundation De Drie Lichten.

Conflict of interest: None.

1 Lensing AWA, Hirsh J, Büller HR. Diagnosis of venous thrombosis. In: Colman RW, Hirsh J, Marder VJ, Salzman EW, eds. Hemostasis and thrombosis: basic principles and clinical practice. 3rd ed. Philadelphia: Lippincott, 1993:1297-321.

2 Lensing AWA, Prandoni P, Büller HR, Casara D, Cogo A, Cate JW. Lower extremity venography with iohexol: results and complications. Radiology 1990;77:503-5.

3 Hull RD, van Aken WG, Hirsh J, Gallus AS, Hoicka G, Turpie AG, et al. Impedance plethysmography using the occlusive cuff technique in the diagnosis of venous thrombosis. Circulation 1976;53:696-700.

4 Hull RD, Hirsh J, Carter CJ, Jay RM, Ockelford PA, Buller HR, et al. Diagnostic efficacy of impedance plethysmography for clinically suspected deep-vein thrombosis: a randomized trial. Ann Intern Med 1985;102:21-6.

5 Hull RD, Raskob GE, Carter CJ. Serial impedance plethysmography in pregnant patients with clinically suspected deep-vein thrombosis. Clinical validity of a negative finding. Ann Intern Med 1990;112:663-7.

6 Huisman MV, Büller HR, ten Cate JW, Vreeken J. Serial impedance plethysmography for suspected deep venous thrombosis in outpatients. The Amsterdam general practitioner study. N Engl J Med 1986;314:823-6.

7 Huisman MV, Büller HR, ten Cate JW, Heijermans HSF, van der Lan J, van Maan en DJ. Management of clinically suspected acute venous thrombosis in outpatients with serial impedance plethysmography in a community hospital setting. Arch Int Med 1989;149:511-5.

8 Heijboer H, Büller HR, Lensing AWA, Turpie AGG, Colly LP, ten Cate JW. A comparison of real-time ultrasonography with impedance plethysmography for the diagnosis of deep-vein thrombosis in symptomatic outpatients. N Engl J Med 1993;329:1365-9.

9 Lensing AWA, Prandoni P, Brandjes D, Huisman PM, Vigo M, Tomasella $\mathrm{G}$, et al. Detection of deep-vein thrombosis by real-time B-mode ultrasonography. N Engl J Med 1989;320:342-5.

10 Appelman PT, de Jong TE, Lampmann LE. Deep venous thrombosis of the leg: US findings. Radiology 1987;163:743-8.

11 Cogo A, Lensing AWA, Prandoni P, Hirsh J. Distribution of thrombosis in patients with symptomatic deep vein thrombosis: implication for simplifying the diagnostic process with compression ultrasound. Arch Intern Med 1993;153:2777-80.

12 Sluzewski M, Koopman MMW, Schuur KH, van Vroonhoven TJMV, Ruijs $\mathrm{JHJ}$. Influence of negative ultrasound findings on the management of inand outpatients with suspected deep-vein thrombosis. Eur J Radiol 1991;13:174-7.

13 Wells PS, Brill-Edwards P, Stevens P, Panju A, Patel A, Douketis J, et al. A novel and rapid whole blood assay for D-dimer in patients with clinically suspected deep vein thrombosis. Circulation 1995;91:2184-7.

14 Wells PS, Hirsh J, Anderson DR, Lensing AWA, Foster G, Kearon C, et al Accuracy of clinical assessment of deep-vein thrombosis. Lancet 1995;345:1326-30.

15 Prandoni P, Lensing AWA, Buller HR, Vigo M, Cogo A, ten Cate JW. Failure of computerized impedance plethysmography in the diagnostic management of patients with clinically suspected deep-vein thrombosis Thromb Haemost 1991;65:233-6.

(Accepted 6 August 1997)

\section{Fifty years ago}

\section{The new NHS: Consultants and the Act}

Such is the confusion of minds and of tongues in the consultant and hospital world that there is unlikely to be any effective challenge to the proposed transfer on July 5, 1948, of the hospitals of Great Britain into the ownership of the State. The implications of this revolutionary step will become more and more visible as the months and the years pass. While many consultants and specialists may accept this as an inevitable step, and some may accept it as a desirable step, we believe that the majority are still opposed to a State Medical Service. In the last plebiscite more than $50 \%$ of consultants and specialists voting voted against negotiations with the Minister under the present Act. (BMJ 1948;i:17) 